\title{
Oscillatory flow around the edge of a flat plate
}

\author{
J. H. M. DISSELHORST AND L. VAN WIJNGAARDEN \\ Department of Applied Physics, Twente University of Technology, Enschede, The Netherlands
}

(Received March 30, 1979)

\section{SUMMARY}

The paper deals with oscillatory flow of an incompressible viscous fluid around the edge of a flat plate. The primary interest is, in connexion with the flow in open pipes near the edge due to acoustic standing waves, in the dissipation associated with the flow around the edge. Mathematically, the problem to find the flow around the edge can be formulated as an integral equation for a dipole distribution along the plate. This can be simplified by making use of the fact that the Stokes boundary layer is thin with respect to the characteristic length scale of the flow. The simplified equation is solved by a method used recently by Boersma. With the help of this solution the dissipation is calculated. The result is compared with exact, numerical, calculation by Disselhorst. Good agreement is found.

\section{Introduction}

In the course of his work on resonant acoustic oscillations in pipes, Disselhorst [1] considers viscous and thermal dissipation associated with these wave motions.
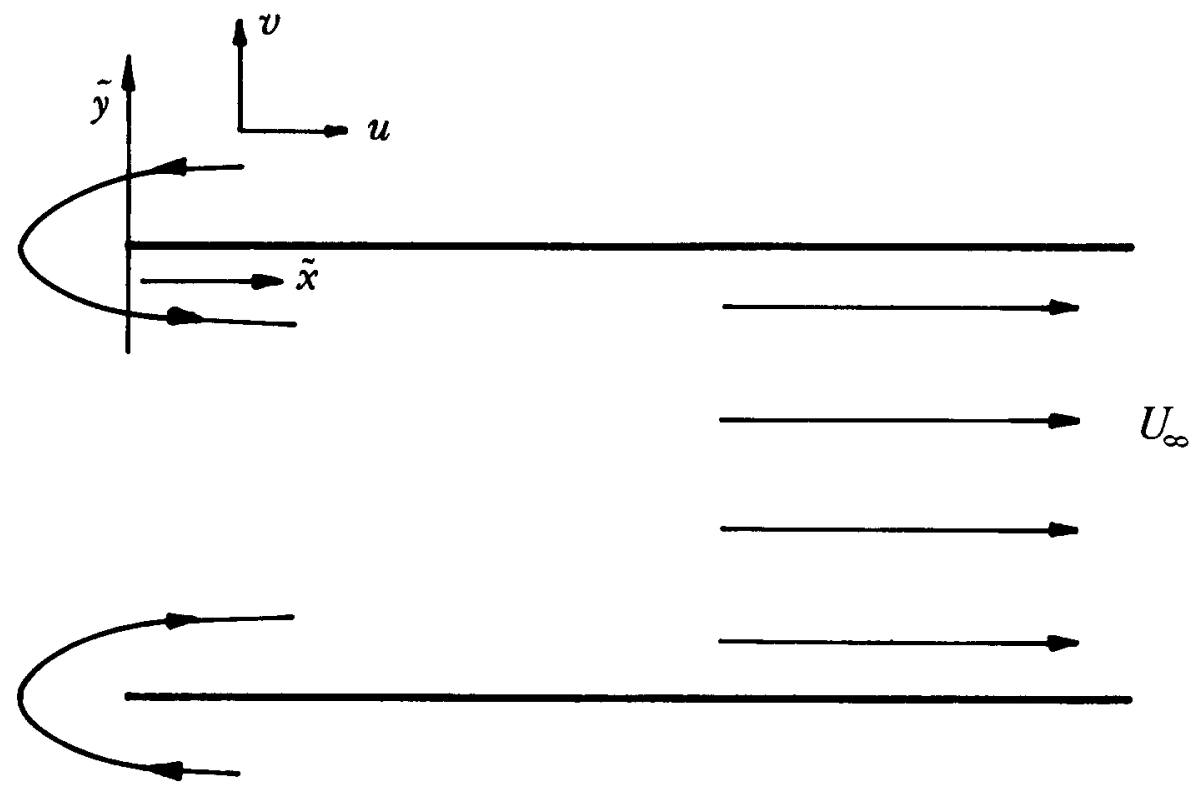

Figure 1. Oscillatory flow around the edge of a pipe or around the edge of a flat plate in the presence of another one. 
The largest part of this takes place in thin boundary layers along the walls of the tube. When the flow does not separate from the edges, that means, when the amplitude of the motion is small*, this dissipation should equal the external work on the air inside the tube. Measurements indicate, however, a dissipation which is systematically by a few percent larger than would follow from dissipation in the boundary layers at the wall. A possible explanation lies in the additional dissipation in the flow around the edge (Figure 1) where strong velocity gradients occur. Disselhorst [1] therefore gives attention to the flow around the edge. This can be considered as locally two-dimensional and this two-dimensional flow may be, for sufficiently low amplitutes, described by the linearized Navier-Stokes equations. Starting from these equations Disselhorst formulates a Wiener-Hopf problem for the stream function. As often, a hard problem is the splitting of the kernel.

This is done in Disselhorst [1] by application of an idea due to Koiter [2], which consists in replacing the kernel by a simpler one with, however, the same behaviour on the real axis. In the final stage the computation is numerical in Disselhorst [1] and results in the viscous dissipation expressed in terms of the corresponding dissipation in the boundary layer approximation (which is singular at $\tilde{x}=0$ in Figure 1), however, not starting at $\tilde{x}=0$ but at $\tilde{x}=\tilde{x}_{0}$. Later also the kernel splitting was done numerically, as reported in Disselhorst [3]. Meanwhile the question may be asked whether a simpler (approximate) solution is possible by making use of the following feature of the flow.

The characteristic length scale of the flow is the diameter of the pipe. The characteristic viscous length in the flow around the edge is $(\nu / \Omega)^{\frac{1}{2}}$, where $\nu$ is the viscosity of air $\left(1.5 \times 10^{-6} \mathrm{~m}^{2} / \mathrm{s}\right)$ and $\Omega$ the angular frequency of the motion (of order $\left.10^{2} \mathrm{c} / \mathrm{s}\right)$. The ratio between them is very small and this allows a simplification which will be carried out in the next section. In Section 3 the resulting integral equation will be solved by using a method of solution applied recently by Boersma [4]. Finally the result for the dissipation is compared with those obtained by Disselhorst [1], [3].

\section{Formulation of the problem}

We consider standing acoustic waves in an open circular tube. The flow is two-dimensional near the edge and like depicted in Figure 1: alternating and flowing around the edge with angular frequency $\Omega$. Because of the two-dimensional character of the flow in the vicinity of the edge we consider here two parallel plates, a distance $2 \pi d$ apart. The viscous flow around the edge of each of the plates may be described for sufficiently low amplitude by the linearized NavierStokes equations which read in terms of the Cartesian coordinates $\tilde{x}$ and $\tilde{y}$ with origin at the edge, and time $t$ (see Figure 1) for the corresponding velocity components $u$ and $v$ and the pressure $p$ :

$$
\begin{aligned}
& \frac{\partial u}{\partial t}=-\frac{1}{\rho} \frac{\partial p}{\partial \tilde{x}}+\nu\left(\frac{\partial^{2} u}{\partial \tilde{x}^{2}}+\frac{\partial^{2} u}{\partial \tilde{y}^{2}}\right), \\
& \frac{\partial v}{\partial t}=-\frac{1}{\rho} \frac{\partial p}{\partial \tilde{y}}+\nu\left(\frac{\partial^{2} v}{\partial \tilde{x}^{2}}+\frac{\partial^{2} v}{\partial \tilde{y}^{2}}\right)
\end{aligned}
$$

* In the application in Disselhorst [1] the edge is round so that for low amplitudes no separation occurs. 


$$
\frac{\partial u}{\partial \tilde{x}}+\frac{\partial v}{\partial \tilde{y}}=0
$$

In formulating the momentum equations (2.1) and (2.2) and the equation for mass conservation (2.3) variations in the density $\rho$ are neglected. This is fully justified by the fact that in the application which we have in mind the wave length is large both compared with $d$ (for a circular tube with radius $R$ the equivalent plate distance is $R$ ) and with $(\nu / \Omega)^{\frac{1}{2}}$ (Disselhorst [1]).

Far from the edge the velocity should approach the inviscid velocity given (with distance $2 \pi d$ between the plates) by

$$
\phi_{0}^{\prime}=\left[U_{\infty}^{2} d\left\{\tilde{x}+\left(\tilde{x}^{2}+\tilde{y}^{2}\right)^{\frac{1}{2}}\right\}\right]^{\frac{1}{2}} e^{i \Omega t} .
$$

In this expression $U_{\infty}$ is the velocity between the plates far from the edges (see Figure 1). In writing down (2.4) it is understood that $\phi_{0}^{\prime}$ is the real part of the expression at the right-hand side. Of course, (2.4) represents the true inviscid velocity only in the vicinity of the edge. The true inviscid velocity tends for $\tilde{x} \rightarrow \infty$ inside the plates to $U_{\infty}$ and outside the plates to zero. Since we are, in this context, only interested in the vicinity of the edge, the approximation (2.4) suffices. At the plate the no-slip condition has to be satisfied,

$$
u=v=0 \quad \text { on } \quad \tilde{y}=0, \quad \tilde{x}>0,
$$

while symmetry requires

$$
u=0 \quad \text { on } \quad \tilde{y}=0, \quad \tilde{x}<0 .
$$

From $(2.1)-(2.3)$ it follows that the pressure $p$ is a harmonic function. When $\phi^{\prime}$ is a solution of Laplace's equation we may therefore write

$$
p=-\rho \frac{\partial \phi^{\prime}}{\partial t}
$$

Without vorticity, $\phi^{\prime}$ would be the velocity potential. With vorticity we can represent the velocity as resulting from $\phi^{\prime}$ and a stream function $\Psi^{\prime}$,

$$
\begin{aligned}
& u=\frac{\partial \phi^{\prime}}{\partial \widetilde{x}}+\frac{\partial \Psi^{\prime}}{\partial \tilde{y}}, \\
& v=\frac{\partial \phi^{\prime}}{\partial \widetilde{y}}-\frac{\partial \Psi^{\prime}}{\partial \widetilde{x}} .
\end{aligned}
$$

Inserting (2.7) - (2.9) into (2.1) and (2.2) gives for the stream function $\Psi^{\prime}$ the equation

$$
\frac{\partial \Psi^{\prime}}{\partial t}=\nu\left(\frac{\partial^{2} \Psi^{\prime}}{\partial \tilde{x}^{2}}+\frac{\partial^{2} \Psi^{\prime}}{\partial \tilde{y}^{2}}\right)
$$


It is convenient to split up the potential $\phi^{\prime}$ in

$$
\phi^{\prime}=\phi_{0}^{\prime}+\phi_{1}^{\prime}
$$

where $\phi_{0}^{\prime}$ is given by (2.4) and represents the velocity field far from the plate outside the region containing the vorticity. The potential $\phi_{1}^{\prime}$ is unknown and must be chosen in such a way that together with $\Psi^{\prime}$ the equation (2.10) and the boundary conditions (2.5) and (2.6) are satisfied. At infinity both $\Psi^{\prime}$ and $\phi_{1}^{\prime}$ must vanish. Associated with $\phi_{1}^{\prime}$ is a stream function $\Psi_{1}^{\prime}$ according to the complex potential $w^{\prime}$,

$$
w^{\prime}=\phi_{1}^{\prime}+i \Psi_{1}^{\prime}
$$

Expressing the irrotational part of the disturbance velocities in $\Psi_{1}^{\prime}$ rather than $\phi_{1}^{\prime}$, we obtain from (2.5), (2.6), (2.8), (2.9) and (2.11), using (2.4),

$$
\begin{aligned}
& \Psi^{1}+\Psi_{1}^{\prime}=0 \quad \text { on } \tilde{y}=0, \quad \tilde{x}>0, \\
& \frac{\partial \Psi^{\prime}}{\partial \tilde{y}}+\frac{\partial \Psi_{1}^{\prime}}{\partial \tilde{y}}+\left(\frac{U_{\infty}^{2} d}{2 \tilde{x}}\right)^{\frac{1}{2}} e^{i \Omega t}=0 \text { on } \tilde{y}=0, \quad \tilde{x}>0, \\
& \frac{\partial \Psi^{\prime}}{\partial \tilde{y}}+\frac{\partial \Psi_{1}^{\prime}}{\partial \tilde{y}}=0, \quad \text { on } \tilde{y}=0 ; \quad \tilde{x}<0 .
\end{aligned}
$$

Finally,

$$
\left.\begin{array}{l}
\Psi_{1}^{\prime} \rightarrow 0 \\
\Psi^{\prime} \rightarrow 0
\end{array}\right\} \text { for }\left(\tilde{x}^{2}+\tilde{y}^{2}\right)^{\frac{1}{2}} \rightarrow \infty
$$

Because of the linearity of the problem, all dependent variables are proportional to $e^{i \Omega t}$. In order to get rid of this factor we introduce

$$
\begin{aligned}
& \Psi^{\prime}=\Psi e^{i \Omega t}, \\
& \Psi_{1}^{\prime}=\Psi_{1} e^{i \Omega t} .
\end{aligned}
$$

Introducing this in Laplace's equation for $\Psi_{1}^{\prime}$ and (2.10) for $\Psi^{\prime}$ we get, with

$$
\lambda^{2}=\frac{i \Omega}{\nu}
$$

for $\Psi$ and $\Psi^{\prime}$ :

$$
\frac{\partial^{2} \Psi}{\partial \widetilde{x}^{2}}+\frac{\partial^{2} \Psi}{\partial \tilde{y}^{2}}-\lambda^{2} \Psi=0,
$$




$$
\frac{\partial^{2} \Psi_{1}}{\partial \widetilde{x}^{2}}+\frac{\partial^{2} \Psi_{1}}{\partial \tilde{y}^{2}}=0
$$

Introduction of (2.16) and (2.17) in (2.12) - (2.15) gives

$$
\begin{aligned}
& \Psi+\Psi_{1}=0 \quad \text { on } \quad \tilde{y}=0, \quad \tilde{x}>0, \\
& \frac{\partial \Psi}{\partial \tilde{y}}+\frac{\partial \Psi_{1}}{\partial \tilde{y}}+\left(\frac{U_{\infty}^{2} d}{2 \tilde{x}}\right)^{\frac{1}{2}}=0 \quad \text { on } \quad \tilde{y}=0, \quad \tilde{x}>0, \\
& \frac{\partial \Psi}{\partial \tilde{y}}+\frac{\partial \Psi_{1}}{\partial \tilde{y}}=0, \quad \text { on } \quad \tilde{y}=0, \quad \tilde{x}<0, \\
& \left.\begin{array}{l}
\Psi \rightarrow 0 \\
\Psi_{1} \rightarrow 0
\end{array}\right\} \text { for }\left(\tilde{x}^{2}+\tilde{y}^{2}\right)^{\frac{1}{2}} \rightarrow \infty .
\end{aligned}
$$

A solution of (2.19) which vanishes at infinity and for which $\partial \Psi / \partial \tilde{y}=0$ on $\tilde{x}<0, \tilde{y}=0$, is

$$
\Psi=\frac{1}{\pi} \int_{0}^{\infty} \tilde{f(\xi)} K_{0}\left[\lambda\left\{(\tilde{x}-\tilde{\xi})^{2}+\tilde{y}\right\}^{\frac{1}{2}}\right] d \tilde{\xi}
$$

where $K_{0}$ is the modified Bessel function of the second kind and $f(x)$ an arbitrary function defined for $x>0$. Likewise, a solution of (2.20) satisfying $\partial \Psi_{1} / \partial \tilde{y}=0$ on $\tilde{y}=0, \widetilde{x}<0$, vanishing at infinity and due to a dipole distribution $g(\tilde{x})$ is

$$
\Psi_{1}=\frac{1}{2 \pi} \int_{0}^{\infty} \frac{g(\tilde{\xi})(\tilde{\xi}-\tilde{x})}{\left.\{\tilde{\xi}-\tilde{x})^{2}+\tilde{y}^{2}\right\}} d \tilde{\xi}
$$

The sum of the right-hand sides of (2.25) and (2.26) has yet to satisfy the boundary conditions (2.21) and (2.22), which yields

$$
\begin{aligned}
& \frac{1}{2 \pi} \int_{0}^{\infty} \frac{g(\tilde{\xi})}{\tilde{\xi}-\tilde{x}} d \tilde{\xi}+\frac{1}{\pi} \int_{0}^{\infty} f(\tilde{\xi}) K_{0}\{\lambda \mid\{\tilde{x}-\tilde{\xi} \mid\} d \tilde{\xi}=0, \tilde{x}>0, \\
& -\frac{1}{2} \frac{d g}{d \tilde{x}}-f(\tilde{x})+\left(\frac{U_{\infty}^{2} d}{2 \tilde{x}}\right)^{\frac{1}{2}}=0 .
\end{aligned}
$$

Elimination of $f(\tilde{x})$ from (2.27) and (2.28) yields

$$
\frac{1}{2} \int_{0}^{\infty} \frac{g(\tilde{\xi})}{\tilde{\xi}-\tilde{x}} d \tilde{\xi}+\int_{0}^{\infty}\left\{\left(\frac{U_{\infty}^{2} d}{2 \tilde{\xi}}\right)^{\frac{1}{2}}-\frac{1}{2} \frac{d g}{d \tilde{\xi}}\right\} K_{0}\{\lambda|\tilde{x}-\tilde{\xi}|\} d \tilde{\xi}=0, \tilde{x}>0
$$


Although the approach in Disselhorst [1] differs from the one presented here one could say that Disselhorst's solution is equivalent to solving the integrodifferential equation (2.29). We now use the fact that in the envisaged application to acoustic waves in tubes $\lambda$, as given in (2.18), is a large quantity. This means that the main contribution to the second integral at the left-hand side of (2.29) comes from the neighbourhood of $\tilde{\xi}=\tilde{x}$. Since

$$
\int_{-\infty}^{\infty} K_{0}\{\lambda|x|\} d x=\frac{\pi}{\lambda}
$$

we may, for sufficiently large $\lambda$, approximate the second integral in (2.29) by

$$
\frac{\pi}{\lambda}\left\{\left(\frac{U_{\infty}^{2} d}{2 \tilde{x}}\right)^{\frac{1}{2}}-\frac{1}{2} \frac{d g}{d \tilde{x}}\right\}
$$

Then the equation (2.29) reduces to

$$
\frac{1}{2 \pi} \int_{0}^{\infty} \frac{g(\tilde{\xi})}{\tilde{\xi}-x} d \tilde{\xi}=\frac{1}{2 \lambda} \frac{d g}{d \tilde{x}}-\frac{1}{\lambda}\left(\frac{U_{\infty}^{2} d}{2 \tilde{x}}\right)^{\frac{1}{2}}, \quad \tilde{x}>0 .
$$

In the remainder of this paper we will be occupied with solution of (2.30).

In order to appreciate the nature of the approximation involved in approximating (2.29) with (2.30), it is illuminating to derive (2.30) in another way. It suggests itself in view of the large value of $(\nu / \Omega)^{\frac{1}{2}}$ in respect to $d$, to take the boundary layer approximation of the equations (2.19) and (2.20), that is, to neglect the derivatives with respect to $\tilde{x}$ and to consider only the region $\tilde{x}>0$. Then, with

$$
\Psi_{0}=\left(\frac{U_{\infty}^{2} d}{2 \hat{x}}\right)^{\frac{1}{2}} \tilde{y}
$$

being the counterpart (for small $\tilde{y}$ ) of $\phi_{0}$, it is readily verified that the 'boundary layer' solutions are

$$
\begin{aligned}
& \Psi_{(B)}=\frac{1}{\lambda}\left(\frac{U_{\infty}^{2} d}{2 \tilde{x}}\right) \exp (-\lambda \tilde{y}), \\
& \Psi_{1(B)}=-\frac{1}{\lambda}\left(\frac{U_{\infty}^{2} d}{2 \tilde{x}}\right)^{\frac{1}{2}},
\end{aligned}
$$

so that the resulting stream function is in $\tilde{y} \geqslant 0$ :

$$
\Psi_{R}=\left(\frac{U_{\infty}^{2} d}{2 \tilde{x}}\right)^{\frac{1}{2}}\left\{\tilde{y}-\frac{1}{\lambda}+\frac{1}{\lambda} \exp (-\lambda \tilde{y})\right\}
$$


The associated vorticity is

$$
\omega=-\nabla^{2} \Psi_{R} \sim-\frac{\partial^{2} \Psi_{R}}{\partial \tilde{y}^{2}}=-\lambda\left(\frac{U_{\infty}^{2} d}{2 \tilde{x}}\right)^{\frac{1}{2}} \exp (-\lambda \tilde{y}) .
$$

The dissipation is

$$
D \sim \int_{0}^{\infty} d \tilde{x} \int_{-\infty}^{\infty}|\omega|^{2} d \tilde{y}
$$

When we insert (2.34) into (2.35) it is readily seen that the integral diverges because of the behaviour of $|\omega|^{2}$ at both $\widetilde{x} \rightarrow 0$ and $\widetilde{x} \rightarrow \infty$. The latter divergence is not serious because it is due to the assumed behaviour of the velocity (derived from $\phi_{0}^{\prime}$ in (2.4)) outside the boundary layer. The former divergence however is due to the boundary-layer approximation, which neglects $\tilde{x}$-derivatives with respect to $\tilde{y}$-derivatives which is not justified near $\tilde{x}=0$. We seek to remedy this by taking the boundary-layer approximation to the rotational part $\Psi$ of the stream function, which means replacing $(2.19)$ by

$$
\frac{\partial^{2} \Psi}{\partial \tilde{y}^{2}}-\lambda^{2} \Psi=0
$$

while keeping (2.20) as it stands. The solution of (2.36) vanishing for $\tilde{y} \rightarrow \infty$ is

$$
\Psi=A(\tilde{x}) \exp (-\lambda \tilde{y})
$$

and a similar one $\sim \exp (\lambda \tilde{y})$ for $\tilde{y} \rightarrow-\infty$. With (2.37) in stead of (2.25) and with (2.26), the conditions (2.21) and (2.22) become

$$
\begin{aligned}
& A(\tilde{x})+\frac{1}{2 \pi} \int_{0}^{\infty} \frac{g(\tilde{\xi})}{\tilde{\xi}-\tilde{x}} d \tilde{\xi}=0, \quad \tilde{x}>0, \\
& -\frac{1}{2} \frac{d g}{d \tilde{x}}-\lambda A(\tilde{x})+\left(\frac{U_{\infty}^{2} d}{2 \tilde{x}}\right)^{\frac{1}{2}}=0 .
\end{aligned}
$$

Eliminiation of $A(\tilde{x})$ between (2.38) and (2.39) gives again equation (2.30). This integral equation is of a type discussed by Van de Vooren and Veldman [5] and solved by Brown [6] and in a particularly elegant way by Boersma [4]. In the following we shall attempt a solution of (2.30) along the lines of Boersma [4]. 


\section{Solution of the integral equation}

We render the spatial coordinate dimensionless by scaling it with the viscous length $\{|\lambda|\}^{-1}=$ $(\nu / \Omega)^{\frac{1}{2}}($ see 2.18$)$,

$$
\widetilde{x}=\frac{x}{2|\lambda|}
$$

Introducing this into (2.30) we get, with the help of (2.18), the following integral equation for $g(x)$ :

$$
\frac{d g}{d x}=\frac{e^{i \pi / 4}}{2 \pi} \int_{0}^{\infty} \frac{g(t)}{t-x} d t+\frac{1}{2} \gamma x^{-\frac{1}{2}}, \quad x>0
$$

where

$$
\gamma=2^{3 / 2}\left(\frac{U_{\infty}^{2} d}{2|\lambda|}\right)^{\frac{1}{2}}
$$

Before proceeding it is, for later reference, useful to determine the asymptotic behaviour of $g(x)$ for large and small $x$. For large values of $x$ the boundary-layer solution explored in the preceding section is fairly accurate. Since

$$
\int_{0}^{\infty} \frac{\ln t}{t^{\frac{1}{2}}(t-x)} d t=\frac{\pi^{2}}{x^{\frac{1}{2}}}, x>0,
$$

it follows from (2.26) and (2.32) that in the boundary-layer approximation

$$
g(x) \sim \frac{\ln x}{x^{\frac{1}{2}}}
$$

This means that $d g / d x \sim \ln x / x^{3 / 2}$ for large $x$ and therefore it is negligibly small with respect to the other terms in (3.2). Putting the right-hand side of (3.2) equal to zero we get, using (3.4),

$$
g(x) \sim-\gamma \pi^{-1} e^{-i \pi / 4} \frac{\ln x}{x^{\frac{1}{2}}}, x \gg 1,
$$

in accordance with (3.5). Near the origin, $x \ll 1$, the term $d g / d x$ in (3.2) dominates over the integral term whence

$$
g(x) \sim \gamma x^{\frac{1}{2}}, x \ll 1
$$

In order to solve (3.2) for $g(x)$ we introduce, following Boersma [4], 


$$
F(z)=-\frac{1}{2 \pi i} \int_{0}^{\infty} \frac{g(t)}{t-z} d t
$$

From (3.6) and (3.7) it follows that $F(z)$ is analytic in the $z$-plane, cut along the positive real axis. When we denote $F(x \pm i o)$ by $F^{ \pm}(x)$ we have from the well-known Plemelj relations (see e.g. Roos [7])

$$
\begin{array}{ll}
F^{+}(x)+F^{-}(x)=-\frac{1}{\pi i} \int_{0}^{\infty} \frac{g(t)}{t-x} d t, \\
F^{+}(x)-F^{-}(x)=-g(x), & x>0, \\
F^{+}(x)-F^{-}(x)=0, & x<0 .
\end{array}
$$

With these relations, the singular integral equation (3.2) can be written as

$$
-\frac{d F^{+}}{d x}+\frac{1}{2} i e^{i \pi / 4} F^{+}=-\frac{d F^{-}}{d x}-\frac{1}{2} i e^{i \pi / 4} F^{-}+\frac{1}{2} \gamma x^{-\frac{1}{2}} .
$$

Now we define, like Boersma [4], the complex Laplace transform $G(s)$ of $F(z)$ by

$$
G(s)=\int_{0}^{\infty} e^{-s z / 2} F(z) d z
$$

with

$$
\arg s+\arg z=0 .
$$

$G(s)$ is analytic in the wole $s$-plane with the exception of the positive real axis $s=\sigma, \sigma>0$, where $G(\sigma)$ has a discontinuity. Indicating $G(\sigma \pm i o)$ by $G^{ \pm}(\sigma)$, we have from (3.7), (3.9), (3.10), (3.12) and (3.13):

$$
\begin{aligned}
& G^{ \pm}(\sigma)=\int_{0}^{\infty} F^{\mp}(x) e^{-\sigma x / 2} d x, \\
& \int_{0}^{\infty} \frac{d F^{\mp}}{d x} e^{-\sigma x / 2} d x=\frac{\sigma}{2} G^{ \pm}(\sigma)+\frac{1}{2 \pi i} \int_{0}^{\infty} \frac{g(t)}{t} d t .
\end{aligned}
$$

With the help of these relations, Laplace transformation of (3.11) gives with

$$
q=\exp (3 \pi i / 4)
$$

the following inhomogeneous Hilbert problem

$$
\begin{gathered}
(\sigma+q) G^{+}(\sigma)-(\sigma-q) G^{-}(\sigma)=\gamma \sqrt{2 \pi} \sigma^{-\frac{1}{2}}, \sigma>0 . \\
G^{+}(\sigma)-G^{-}(\sigma)=0, \sigma<0 .
\end{gathered}
$$


In the following solution we define the branch cut of $s^{\frac{1}{2}}$ and of $\ln s$ on the negative real axis. The solution of this Hilbert problem can be achieved in a slightly different way compared with the solution in [4] because in this particular case we observe that

$$
(\sigma+q)\left[\frac{\gamma \sqrt{2 \pi}}{2 s i(-s)^{\frac{1}{2}}}\right]_{s=\sigma+i o}-(\sigma-q)\left[\frac{\gamma \sqrt{2 \pi}}{2 s i(-s)^{\frac{1}{2}}}\right]_{s=\sigma-i o}=\frac{\gamma \sqrt{2 \pi}}{\sigma^{\frac{1}{2}}} .
$$

This means that if $X(s)$ is the solution of the corresponding homogeneous Hilbert problem, defined by

$$
\begin{array}{rr}
(\sigma+q) X^{+}(\sigma)-(\sigma-q) X(\sigma)=0, & \sigma>0, \\
X^{+}(\sigma)-X^{-}(\sigma)=0, & \sigma<0,
\end{array}
$$

the solution of $(3.17)$ is

$$
G(s)=\frac{(2 \pi)^{\frac{1}{2}} \gamma}{2 i s(-s)^{\frac{1}{2}}}+P(s) X(s)
$$

$P(s)$ being an arbitrary entire function of $s$. The solution of $(3.18)$ is

$$
\ln X(s)=W(s)=\frac{1}{2 \pi i} \int_{0}^{\infty} \frac{\ln \{(t-q) /(t+q)\}}{t-s} d t
$$

From this it follows

$$
\begin{aligned}
\frac{d W(s)}{d s}= & -\frac{1}{2 \pi i} \int_{0}^{\infty} \ln \frac{t-q}{t+q} \frac{d}{d t} \frac{1}{t-s} d t= \\
& -\frac{1}{2 \pi i} \frac{1}{s} \ln \frac{-q}{q}+\frac{1}{s-q} \ln \frac{-s}{-q}-\frac{1}{s+q} \ln \frac{-s}{q} .
\end{aligned}
$$

We obtain by integration of the above relation

$$
W(s)=\ln (-s)^{\frac{1}{2}}-\ln (s-q)^{\frac{1}{4}}-\ln (s+q)^{\frac{1}{4}}-h(s),
$$

with

$$
h(s)=-\frac{1}{2 \pi i} \int_{0}^{\infty}\left(\frac{1}{z+q}-\frac{1}{z-q}\right)\left\{\ln (-z)-\frac{\pi i}{4}\right\} d z
$$

Since (cf. 3.20) $W(s)=\ln (X(s))$ we get from (3.21) and (3.22) for the solution of the homogeneous Hilbert problem (3.18), 


$$
X(s)=\frac{(-s)^{\frac{1}{2}}}{\{(s-q)(s+q)\}^{\frac{1}{4}}} e^{-h(s)} .
$$

By inserting (3.23) in (3.19) we may obtain an expression for $G(s)$. Eventually we are interested in $g(x)$, the Laplace transform of which, $M(s)$, say, is given by, from (3.10) and (3.14),

$$
\begin{aligned}
M(\sigma)=\int_{0}^{\infty} e^{-\sigma x / 2}\left\{F^{(}(x)-F^{+}(x)\right\} d x & =\int_{0}^{\infty} g(x) e^{-\sigma x / 2} d x \\
& =G^{+}(\sigma)-G^{-}(\sigma) .
\end{aligned}
$$

From this relation and from (3.19) it follows that we need to determine the behaviour $X^{\ddagger}(\sigma)$ of $X(s)$ for $s=\sigma \pm i o$. From (3.22) and (3.23) we have, defining $H(s)$ as

$$
\begin{aligned}
& H(s)=-\frac{1}{2 \pi i} \int_{0}^{s}\left(\ln t-\frac{\pi i}{4}\right)\left(\frac{1}{t+q}-\frac{1}{t-q}\right) d t \\
& X^{ \pm}(\sigma)=-\frac{\sigma^{\frac{1}{2}}}{(\sigma-q)^{\frac{1}{4}}(\sigma+q)^{\frac{1}{4}}} \frac{(\sigma \mp q)^{\frac{1}{2}}}{(\sigma \pm q)^{\frac{1}{2}}} e^{-H(\sigma)} .
\end{aligned}
$$

With the help of (3.19) and (3.26) we find for $M(\sigma)$ as related to $G(s)$ by (3.24),

$$
M(\sigma)=\frac{\gamma \sqrt{2 \pi}}{\sigma^{3 / 2}}-P(\sigma) \frac{2 q \sigma^{1 / 2} e^{-H(\sigma)}}{(\sigma-q)^{3 / 4}(\sigma+q)^{3 / 4}} .
$$

The unknown entire function $P(s)$ has to be determined from the following conditions to be imposed on $M(\sigma)$ :

i) The asymptotic behaviour of $g(x)$, as expressed in (3.6) for large $x$ and in (3.7) for small $x$, leads with the help of the theory of Laplace transformations, see e.g. Carslaw and Jaeger [8], to conditions for the asymptotic behaviour of $M(\sigma)$. Since for large $x, g(x)$ behaves as $x^{-\frac{1}{2}} \ln x$, $M(\sigma)$ must for small $\sigma$ behave as the Laplace transform of $x^{-\frac{1}{2}} \ln x$ which behaves, see e.g. Campbell and Foster [9], as $\sigma^{-\frac{1}{2}} \ln \sigma$. Therefore, from (3.6),

$$
M(\sigma) \sim \pi^{-1}(2 \pi)^{\frac{1}{2}} e^{-i \pi / 4} \frac{\ln \sigma}{\sigma^{\frac{1}{2}}}, \sigma \rightarrow 0 .
$$

Likewise it follows from (3.7) that

$$
M(\sigma) \sim \frac{\gamma(2 \pi)^{\frac{1}{2}}}{\sigma^{3 / 2}}, \sigma \rightarrow \infty
$$

ii) When $M(\sigma)$ is inverted by use of the inversion theorem of the Laplace transformation, it follows from $g(x)=0$ for $x<0$ that $M(s)$, the analytic continuation of $M(\sigma)$ in the (complex) splane cannot have singularities in the right-half plane.

From (3.25) and (3.27) we obtain for $\sigma \rightarrow 0$ : 


$$
M(\sigma) \sim \frac{\gamma(2 \pi)^{\frac{1}{2}}}{\sigma^{3 / 2}}-2 P(\sigma) e^{3 \pi i / 8} \sigma^{\frac{1}{2}}\left(1+\frac{\sigma \ln \sigma}{i \pi q}\right) .
$$

Comparison with (3.28) shows that therefore

$$
2 P(\sigma) e^{3 \pi i / 8}=\frac{(2 \pi)^{\frac{1}{2}} \gamma}{\sigma^{2}}\left(1+a_{1} \sigma+a_{2} \sigma^{2}+\ldots\right) .
$$

Inserting this in (3.27) and taking account of (3.29) we observe, bearing in mind that $e^{-H(\infty)}$ is finite, that in the brackets in the right-hand side of (3.30) only the first and the second term can be retained. Hence

$$
H(\sigma)=\frac{\gamma(2 \pi)^{\frac{1}{2}}}{\sigma^{3 / 2}}\left\{1-\frac{e^{3 \pi i / 8} e^{-H(\sigma)}}{(\sigma-q)^{3 / 4}(\sigma+q)^{3 / 4}}\left(1+a_{1} \sigma\right)\right\} .
$$

Because of condition ii), formulated above, a singularity of $M(s)$ in $s=-q$ (which is, see (3.16), in the right-half plane) has to be ruled out. In this connexion it is convenient to split up $H(s)$ in a part regular in $s=-q$ and a part which is singular there. This is accomplished by writing instead of (3.25)

$$
\begin{aligned}
H(s) & =-\frac{1}{2 \pi i} \int_{0}^{\infty}\left(\ln t+\frac{\pi i}{4}\right)\left(\frac{1}{t+q}-\frac{1}{t-q}\right) d t+\ln \left(\frac{q+s}{q-s}\right)^{\frac{1}{4}} \\
& =K(s)+\ln \left(\frac{q+s}{q-s}\right)^{\frac{1}{4}},
\end{aligned}
$$

in which the function $K(s)$ is now regular in $s=-q$. Inserting (3.32) in (3.31) we obtain

$$
M(s)=\frac{\gamma(2 \pi)^{\frac{1}{2}}}{s^{3 / 2}}\left\{1-\frac{e^{5 \pi i / 8} e^{-K(s)}}{(s+q)(s-q)^{\frac{1}{2}}}\left(1+a_{1} s\right)\right\}
$$

For this expression to be regular in $s=-q, a_{1}$ has to assume the value $a_{1}=q^{-1}=\exp (-3 \pi i / 4)$, whence we have finally

$$
M(s)=\frac{\gamma(2 \pi)^{\frac{1}{2}}}{s^{3 / 2}}\left\{1-\frac{e^{-\pi i / 8} e^{-K(s)}}{(s-q)^{\frac{1}{2}}}\right\} .
$$

\section{Calculation of dissipation}

If we were interested in the velocity distribution around the edges in Figure 1, we would have to invert $M(s)$, as given in (3.34), which is not possible analytically. As explained in Sections 1 and 2 , our purpose is to calculate the excess dissipation at the edge. If $\omega^{\prime}$ denotes the vorticity, that dissipation is given by 


$$
D=\mu \int_{-\infty}^{-\infty} d \tilde{x} \int_{-\infty}^{-\infty} \overline{\omega^{\prime 2}} d \tilde{y}
$$

where $\mu$ is the dynamic viscosity of the fluid and the bar over $\omega^{\prime 2}$ means the average over a period.

In our theory the boundary-layer approximation has been taken of the vorticity equation (2.10), which means apart from the neglect of the $\tilde{x}$-derivative, that only the positive part of the real axis is considered. When, like with the other quantities, we write

$$
\omega^{\prime}=\omega \exp (i \Omega t)
$$

and use the relation

$$
\omega^{\prime}=-\nabla^{2} \Psi^{\prime}
$$

we can write instead of (4.1):

$$
D=\frac{\mu}{2} \int_{-\infty}^{\infty} d \tilde{x} \int_{-\infty}^{\infty}|\omega|^{2} d \tilde{y}=\frac{\mu}{2} \int_{-\infty}^{\infty} d \tilde{x} \int_{-\infty}^{\infty}\left|\nabla^{2} \Psi\right|^{2} d \tilde{y}
$$

The boundary-layer approximation for the vorticity leads, as shown in Section 2, to (2.36) for $\Psi$, with solution given by (2.37). Inserting this in (4.4) we get

$$
D=\mu \int_{0}^{\infty} d \tilde{x} \int_{0}^{\infty}\left|\lambda^{2} A(\tilde{x}) \exp (-\lambda \tilde{y})\right|^{2} d \tilde{y}
$$

Carrying out the integration with respect to $\tilde{y}$ and using $x$ instead of $\tilde{x}$, as given in (3.1), we find

$$
D=\frac{\mu|\lambda|^{2}}{2^{3 / 2}} \int_{0}^{\infty} A(x) \overline{A(x)} d x,
$$

where we denote the complex conjugate of $A(x)$ by $\overline{A(x)}$. From (2.39), (3.1) and (3.3) we have

$$
A(x)=\frac{|\lambda|}{\lambda}\left\{-\frac{d g}{d x}+\frac{1}{2} \gamma x^{-\frac{1}{2}}\right\}
$$

which may be introduced in (4.5) to give the dissipation $D$. As is readiliy verified the same expression for $D$ is obtained when we start with the solution (2.25) for $\Psi$ and carry out the approximation leading to (2.30). The function $f(\tilde{x})$ is related to $A(\tilde{x})$ by $\lambda A(\tilde{x})=f(\tilde{x})$, as follows from comparison of (2.28) with (2.39). In Section 2 we emphasized that taking the boundary-layer approximation of all equations leads to a divergent integral for the dissipation. In fact, when for $\Psi$ in (4.4) the expression $\Psi_{R}$ in (2.33) is inserted the result is for $D_{B}$ with which we indicate the dissipation in the 'full' boundary-layer approximation,

$$
D_{B}=\frac{\rho U_{\infty}^{2} d(\Omega v)^{\frac{1}{2}}}{2^{3 / 2}} \int_{0}^{\infty} \frac{d x}{x}
$$


This integral diverges both for small and large $x$.

Because of (3.7), $A(x)$ tends to zero for small $x$, leaving only the divergence at $x \rightarrow \infty$ in (4.5). This is, as mentioned in Section 2, due to the behaviour for large $\tilde{x}$ of $\partial \phi_{0}^{\prime} / \partial \tilde{x}$ in (2.4). We could avoid this by choosing instead of $\phi_{0}^{\prime}$ in (2.4) a more complicated expression which for small $x$ behaves like the right-hand side of (2.4) but tends to zero for large $x$. A convergent integral is obtained in another way when we compare the dissipation $D$ as given by (4.5) with the boundary-layer dissipation $D_{B}$ in (4.7), however, not integrated between 0 and $\infty$ but between a value $x_{0}$ and infinity. In this way, used in Disselhorst [1], the true dissipation is expressed as an equivalent portion of $D_{B}$. Hence we write, following Disselhorst [1],

$$
\frac{\mu|\lambda|^{2}}{2^{3 / 2}} \int_{0}^{\infty}\{A(x) \overline{A(x)}\} d x-\frac{\rho U_{\infty}^{2} d(\Omega \nu)^{\frac{1}{2}}}{2^{3 / 2}} \int_{x_{0}}^{\infty} \frac{d x}{x}=0
$$

or, introducing Heaviside's step function $E(x)$ with

$$
\begin{aligned}
& E(x)=0, \quad x<0 \\
& E(x)=1, \quad x>0, \\
& \rho U_{\infty}^{2} d(\Omega \nu)^{\frac{1}{2}} \ln \left(1 / x_{0}\right)=\int_{0}^{\infty}\left\{\mu|\lambda|^{2} A(x) \overline{A(x)}-\frac{\rho U_{\infty}^{2} d(\Omega \nu)^{\frac{1}{2}} E(x-1)}{x}\right\} d x .
\end{aligned}
$$

Using the definition (3.3) for $\gamma$ this can be written as

$$
\gamma^{2} / 4 \ln \left(1 / x_{0}\right)=\int_{0}^{\infty}\left[A(x) \overline{A(x)}-\left\{\frac{\gamma}{2 \sqrt{x}} E(x-1)\right\}^{2}\right] d x
$$

This can, by Parseval's Theorem, (see e.g. Doetsch [10]), be put in the form of an integral of the moduli of the Laplace transforms. The Laplace transform of $A(x)$ can with the help of (3.24) and (4.6) be expressed in terms of $M(s)$ in (3.34). Denoting the Laplace transform of $A(x)$ with $N(s)$ we have from these relations

$$
N(s)=\gamma\left(\frac{\pi}{2}\right)^{\frac{1}{2}} \frac{e^{\pi i / 8} e^{-K(s)}}{s^{\frac{1}{2}}(s-q)^{\frac{1}{2}}} .
$$

When we define $V(\sigma)$ by

$$
\int_{0}^{\infty} e^{-\sigma x / 2} \frac{\gamma}{2 \sqrt{x}} E(x-1) d x=V(\sigma)
$$

we have from (4.10) - (4.12), by virtue of Parseval's Theorem:

$$
\gamma^{2} / 4 \ln \left(1 / x_{0}\right)=\frac{1}{4 \pi} \int_{-\infty}^{\infty}\left\{|N(i \tau)|^{2}-|V(i \tau)|^{2}\right\} d \tau
$$


From (4.11) it follows that

$$
|N(i \tau)|^{2}=N(i \tau) \overline{N(i \tau)}=\frac{\pi \gamma^{2}}{2} \frac{\exp [-2 R\{K(i \tau)\}]}{|\tau|\left\{\tau^{2}-2^{\frac{1}{2}} \tau+1\right\}^{\frac{1}{2}}},
$$

where $R\{\}$ means 'real part of \{\} '. For $\tau>0$ it follows from the definition of $K(s)$ in (3.32), that

$$
-2 R\{K(i \tau)\}=-\frac{2^{\frac{1}{2}}}{\pi} \int_{0}^{\tau} \frac{1+t^{2}}{1+t^{4}} \ln t d t+\frac{3}{8} \ln \frac{\tau^{2}+2^{\frac{1}{2}} \tau+1}{\tau^{2}-2^{\frac{1}{2}} \tau+1} .
$$

Likewise, for $\tau<0$,

$$
-2 R\{K(-i|\tau|)\}=\frac{2^{\frac{1}{2}}}{\pi} \int_{0}^{|\tau|} \frac{1+t^{2}}{1+t^{4}} \ln t d t-\frac{1}{8} \ln \frac{\tau^{2}-2^{\frac{1}{2}}|\tau|+1}{\tau^{2}+2^{\frac{1}{2}}|\tau|+1} .
$$

Evaluation of (4.12) gives

$$
\begin{aligned}
& V(i \tau)=\int_{1}^{\infty} e^{-i \tau x / 2} \frac{\gamma}{\sqrt{2 x}} d x= \\
& \gamma\left(\frac{\pi}{\tau}\right)^{\frac{1}{2}}\left[\frac{1}{2}-C\left(\left|\frac{\tau}{\pi}\right|^{\frac{1}{2}}\right)+i \operatorname{sgn} \tau\left\{\frac{1}{2}-S\left(\left|\frac{\tau}{\pi}\right|^{\frac{1}{2}}\right)\right\}\right]
\end{aligned}
$$

In this expression

$$
\begin{aligned}
& C(x)=\int_{0}^{x} \cos \left(\frac{1}{2} \pi t^{2}\right) d t, \\
& S(x)=\int_{0}^{x} \sin \left(\frac{1}{2} \pi t^{2}\right) d t .
\end{aligned}
$$

Insertion of $(4.14)-(4.17)$ in (4.13) gives for $\ln \left(1 / x_{0}\right)$ :

$$
\begin{aligned}
\ln \left(1 / x_{0}\right)= & \frac{1}{2} \int_{0}^{\infty}\left\{Z(\tau)+\frac{1}{Z(\tau)}\right\} \frac{d \tau}{\left\{\tau^{2}-2^{\frac{1}{2}} \tau+1\right\}^{\frac{1}{2}} \tau} \\
& -\int_{0}^{\infty}\left[1+2 C^{2}\left\{\left(\frac{\tau}{\pi}\right)^{\frac{1}{2}}\right\}+2 S^{2}\left\{\left(\frac{\tau}{\pi}\right)^{\frac{1}{2}}\right\}-2 C\left\{\left(\frac{\tau}{\pi}\right)^{\frac{1}{2}}\right\}\right. \\
& \left.-2 S\left\{\left(\frac{\tau}{\pi}\right)^{\frac{1}{2}}\right\}\right] \frac{d \tau}{\tau} .
\end{aligned}
$$

The function $Z(\tau)$ is defined by

$$
Z(t)=\left\{\frac{t^{2}+2^{\frac{1}{2}} t+1}{t^{2}-2^{\frac{1}{2}} t+1}\right\}^{3 / s} \exp \left\{-\frac{2^{\frac{1}{2}}}{\pi} \int_{0}^{t} \frac{1+y^{2}}{1+y^{4}} \ln y d y\right\}
$$


It is easily verified from the above expression that $Z(t)=Z\left(t^{-1}\right)$. Making use of this property of $Z(t)$ we write (4.20), for numerical purposes, as

$$
\begin{aligned}
\ln \left(1 / x_{0}\right)= & \int_{0}^{1}\left[\frac{1}{2}\left\{Z(\tau)+\frac{1}{Z(\tau)}\right\} \frac{1+\tau}{\left\{1-2^{\frac{1}{2}} \tau+\tau^{2}\right\}^{\frac{1}{2}}}-1\right] \frac{d \tau}{\tau} \\
& +2 \int_{0}^{1}\left[C\left\{\left(\frac{\tau}{\pi}\right)^{\frac{1}{2}}\right\}-C^{2}\left\{\left(\frac{\tau}{\pi}\right)^{\frac{1}{2}}\right\}+S\left\{\left(\frac{\tau}{\pi}\right)^{\frac{1}{2}}\right\}-S^{2}\left\{\left(\frac{\tau}{\pi}\right)^{\frac{1}{2}}\right\}\right. \\
& \left.-\left\{\frac{1}{2}-C\left\{\left(\frac{1}{\pi \tau}\right)^{\frac{1}{2}}\right\}\right\}^{2}-\left\{\frac{1}{2}-S\left\{\left(\frac{1}{\pi \tau}\right)^{\frac{1}{2}}\right\}\right\}^{2}\right] \frac{d \tau}{\tau}
\end{aligned}
$$

The integrand in the first integral at the right-hand side of (4.22) behaves for $\tau \rightarrow 0$ like $1+2^{-1 / 2}+O(\tau)$, the integrand in the second integral as $(\pi \tau)^{-\frac{1}{2}}+O\left(\tau^{\frac{1}{2}}\right)$. Therefore both integrals exist. The integration was carried out numerically and resulted in

$$
\ln \left(1 / x_{0}\right)=4.63
$$

correct in two decimal places. The meaning of this result was set out at the beginning of this section: apart from a constant, $\left\{\rho U_{\infty}^{2} d(\Omega \nu)^{\frac{1}{2}}\right\} / 2^{3 / 2}, \ln \left(1 / x_{0}\right)$ gives (see (4.7)), the dissipation between $x=x_{0}$ and $x=1$ in the most simple kind of boundary-layer theory. The actual dissipation as given by the first term on the left-hand side of (4.8) can be expressed in terms of this, namely as the dissipation according to simple boundary-layer theory, however, not starting in $x=0$ (which would make the dissipation infinitely large) but in $x_{0}$.

Finally we compare our result with the results obtained by Disselhorst. In Disselhorst [1] the only approximation consists of replacing the kernel in the integral equation, according to Koiter's [2] suggestion, by a simpler one. Disselhorst's [1] dimensionless abscis, $\zeta$, say, is related to ours by

$$
\zeta=\frac{1}{2} x
$$

Hence Disselhorst's [1, p. 82] result

$$
2^{\frac{1}{2}} \ln \left(1 / \zeta_{0}\right)=6
$$

gives, written in the variable $x$ used here,

$$
\ln \left(1 / x_{0}\right)=3.55
$$

which is not too far from the result in (4.23). Later an exact numerical calculation of $\ln \left(1 / x_{0}\right)$ was given in Disselhorst [3], resulting in

$$
\ln \left(1 / x_{0}\right)=3.73
$$


Comparison of (4.23) with (4.25) shows that in the approximation which underlies (4.23) the dissipation is a little bit overestimated.

\section{REFERENCES}

[1] J. H. M. Disselhorst. Acoustic resonance in open tubes. Thesis, Twente University of Technology 1978.

[2] W. T. Koiter. Approximate solution of Wiener-Hopf type integral equations with applications. Proc. Kon. Ned. Ak. Wet. B57 (1954) 558.

[3] J. H. M. Disselhorst. To be published.

[4] J. Boersma. Note on an integral equation of viscous flow theory. J. Engineering Mathematics 12 (1978) 237-243.

[5] A. I. van de Vooren and A E. P. Veldman. Incompressible viscous flow near the leading edge of a flat plate admit ting slip. J. Engineering Mathematics 9 (1975) 235-249.

[6] S. N. Brown. On an integral equation of viscous flow theory. J. Engineering Mathematics 11 (1977) 219-226.

[7] B. W. Roos. Analytic functions and distributions in physics and engineering. Wiley \& Sons, New York (1969).

[8] H. S. Carslaw \& J. C. Jaeger. Operational methods in applied mathematics. Oxford University Press (1951).

[9] G. A. Campbell \& R. M. Forster. Fourier integrals for practical applications. Van Nostrand, New York (1948).

[10] G. Doetsch. Introduction to the theory and application of the Laplace transformation. Springer, Berlin (1974). 